\title{
The relation between young children's physiological arousal and their motivation to help others
}

\author{
Robert Hepach $^{\mathrm{a}, *}$, Amrisha Vaish ${ }^{\mathrm{b}}$, Katharina Müller ${ }^{\mathrm{a}}$, Michael Tomasello ${ }^{\mathrm{c}, \mathrm{d}}$ \\ a Leipzig University, Leipzig, Germany \\ ${ }^{\mathrm{b}}$ University of Virginia, Charlottesville, USA \\ ${ }^{\mathrm{c}}$ Duke University, Durham, USA \\ ${ }^{\mathrm{d}}$ Max Planck Institute for Evolutionary Anthropology, Leipzig, Germany
}

\section{A R T I C L E I N F O}

\section{Keywords:}

Children

Prosocial behaviour

Internal arousal

Pupil dilation

Physiology

\begin{abstract}
A B S T R A C T
Children are motivated to help others from an early age. However, little is known about the internal biological mechanisms underlying their motivation to help. Here, we compiled data from five separate studies in which children, ranging in age from 18 months to 5.5 years, witnessed an adult needing help. In all studies, we assessed both (1) children's internal physiological arousal via changes in their pupil dilation, and (2) the latency and likelihood of them providing help. The results showed that the greater the baseline-corrected change in children's internal arousal in response to witnessing the need situation, the faster and more likely children were to help the adult. This was not the case for the baseline measure of children's tonic arousal state. Together, these results suggest that children's propensity to help is systematically related to their physiological arousal after they witness others needing help. This sheds new light on the biological mechanisms underlying not only young children's social perception but also their prosocial motivation more generally.
\end{abstract}

From an early age children attend closely to not only their physical but also their social environment (Grossmann and Johnson, 2007). Infants form expectations on the basis of others' goal-directed behaviour (Reid et al., 2009; Woodward, 2009). This sensitivity includes situations in which agents demonstrate a need in order to fulfill a goal, whereupon infants expect the need to be fulfilled appropriately (Köster et al., 2016; see also Hepach et al., 2016a). Toddlers not only form expectations about others' goal-directed and need-elicited behaviour but also engage and act prosocially to fulfill others' needs (see Eisenberg and Spinrad, 2014; Martin and Olson, 2015; Paulus, 2014; Warneken, 2015, for reviews).

Prosocial behaviour emerges during the first two years of life (Dunfield, 2014; Rheingold and Hay, 1980; Warneken and Tomasello, 2009; Zahn-Waxler et al., 1992). Despite the accumulating empirical evidence documenting helping in young children, an equally robust finding is that the degree of helping varies considerably. When directly comparing rates of helping in different situations, such as comforting, instrumental helping, and sharing, studies document: (a) substantial variation across age groups, (b) variability between experimental tasks, and (c) that sometimes children do not help (Dunfield et al., 2011; Dunfield and Kuhlmeier, 2013; Svetlova et al., 2010). Given the significance of prosocial behaviour for the functioning of social groups, it is crucial to explain these individual differences in children's motivation to help (Eisenberg and Spinrad, 2014).

Prosocial behaviour is embedded in and influenced by the social contexts children grow up in (e.g., Kasten, 2007; Sarafino, 1985; Schmidt-Denter, 2005). Children who visit daycare show more advanced social development than children in homecare (e.g. ClarkeStewart, 2010). In addition, districts with a high concentration of adults having high social status provide more positive role models for children and adolescents including helping (Jencks and Mayer, 1990; Helbig, 2010; Lenzi et al., 2012). Therefore, the societal context children live and grow up in can influence their prosocial behaviour (e.g. Romano et al., 2005). For instance, children with siblings show more prosocial behaviour than firstborns or single children (e.g., Harper et al., 2014). In addition, different parenting styles have been linked to societal milieu and influence the development of prosocial behaviour (Liebenwein, 2008; Zahn-Waxler et al., 1979; see also Brooks-Gunn et al., 1993, for effects of neighborhood on children's development).

On a psychological level, from as early as 12 months of age, children express sympathy and provide help when others show signs of emotional distress (Bischof-Köhler, 1991; Roth-Hanania et al., 2011; ZahnWaxler et al., 1992). In older children, such sympathetic responses have been linked to physiological changes in response to emotional videos

\footnotetext{
* Correspondence to: Department of Research Methods in Early Child Development, Faculty of Education, Leipzig University, Room 107, Jahnallee 59, 04109 Leipzig, Germany.

E-mail address: robert.hepach@uni-leipzig.de (R. Hepach).
} 
displaying others in need (e.g., Eisenberg et al., 1988a). When shown sympathy-inducing videos and given the opportunity to help in a separate context, children with greater heart rate deceleration demonstrated increased helping behaviour, such as packing crayons for a child in need or giving up recess time (Eisenberg et al., 1988b, 1989; Eisenberg and Fabes, 1990). On the other hand, children with increased arousal (heart rate or skin conductance) - also referred to as personal distress - showed less helping behaviour (Eisenberg et al., 1988b; Miller et al., 1996). However, prior empirical findings regarding children's physiological responsiveness and their prosocial behaviour are mixed given that their relation has thus far only been studied indirectly by measuring the physiological variable in one situation and assessing helping in a separate context (Hastings et al., 2006). This is likely due to the methodological challenge of concurrently assessing children's live physiological responses and their helping behaviour within the same helping situation.

In contrast to the majority of previous studies, a recent line of work has more directly measured children's physiological responses to others in need via pupil dilation (Hepach et al., 2012, 2016b). Systematic changes in pupil size reflect changes in internal arousal (Loewenfeld, 1993; Bradley et al., 2008, see Hepach and Westermann, 2016; Laeng, Sirois, and Gredebäck, 2012; Sirois and Brisson, 2014, for recent reviews on pupillometry). When children observed an individual in need, their internal arousal was similar whether they themselves helped or a third party helped and both differed from when the needy person received no help (Hepach et al., 2012). On the other hand, when children had themselves accidentally caused an adult to need help, their internal arousal remained high if they could not themselves reconcile and help with repairing the damage (Hepach et al., 2016b) suggesting that young children were intrinsically motivated to see others' needs fulfilled (see also Warneken and Tomasello, 2008; Hepach et al., 2013; 2016a)."

The focus of this line of work has been on the change in children's pupil dilation from before to after the person in need received help rather than focussing on their tonic arousal state (averaged pupil diameter over time) measured at only one time point. However, whether or not changes in children's internal arousal reflect their prosocial motivation to help crucially depends on whether the degree of children's pupil dilation in response to seeing others in need is predictive of children's subsequent helping behaviour. Some evidence suggests that this is the case. In one previous analysis, children's process tonic pupil dilation in response to seeing an adult needing help was negatively correlated with their latency to subsequently provide help (Hepach et al., 2013). In a similar analysis, children's process tonic pupil dilation but not their baseline pupil dilation measured at the beginning of the test trial was negatively related to how fast they helped (Hepach et al., 2016). Though suggestive, it is important to note that both analyses were carried out on data from only one age group (24-month-old children) and did not specifically analyze the change in children's pupil dilation from baseline to after they saw the other individual needing help. This baseline-corrected change in arousal would be a stronger indication for a change in children's motivation than an assessment of only their arousal state before they helped. In the context of studying the underlying motivation of behaviour such changes in internal arousal reflect the strength or force that drives an agent to attain his or her goal (Brehm and Self, 1989; Richter et al., 2016; Wright and Kirby, 2001). The crucial question thus is whether this triggered "motivation arousal" (Brehm and Self, 1989) reflects how much children want to help an agent attain his goal or to fulfill her need. In other words, does the change in children's arousal predict whether and how fast they will subsequently help?

To address the question of whether changes in internal arousal (assessed via changes children's pupil dilation) reflect motivation, we compiled data comprising four different age groups of children (18 months, 24 months, 3 years, and 5 years) as they witnessed an adult needing help with an instrumental task in various contexts. The data are drawn from control conditions of five studies either published or submitted elsewhere. We did not include the experimental conditions from those studies because they all varied different aspects of the helping situation, both before and after children provided help. The exception are the data from an experiment reported in Hepach et al. (2016a) which are fully included in the present analyses (see Methods for details).

In all cases, children's pupil dilation was measured at two time points: prior to witnessing the adult needing help (baseline measure) and immediately after witnessing the need situation (process measure). We then calculated the baseline-corrected change in children's pupil dilation: (process - baseline measure) / baseline measure. Children were subsequently given the opportunity to help and data were collected on whether they helped and how fast they were to do so. If changes in pupil dilation do indeed reflect children's motivation to help others, we expected a positive relation between the change in children's pupil dilation in response to seeing the adult needing help and the likelihood that they would provide help. On the other hand, we predicted no such relation with regards to children's baseline measure of pupil dilation (see also Hepach et al., 2016ba). We explored individual differences with regards to the social context on two levels: children's helping behaviour and the change in children's pupil dilation in response to seeing help needed. We included control variables from the wider social context (siblings, daycare, and societal) given their previously shown influence on children's prosocial behaviour (e.g., Dunn and Munn, 1986; Howes and Rubenstein, 1981; Sarafino, 1985; see Hastings et al., 2005, for a review) in addition to controlling for gender and children's age. At the same time, the focus of our analyses was the relation between children's change in pupil dilation and their subsequent helping behaviour. In the discussion, we address the implications of the above-mentioned control variables to study the broader context in which children's prosocial motivation develops.

\section{Methods}

\subsection{Participants}

The data comprised 109 children (54 girls) drawn from five separate samples. Twenty-two 1.5-year-old children ( 10 boys; Median $=1$ year 6 months 28 days; Range $=1$ year 6 months 9 days to 1 year 8 months 25 days), a second sample of 22 2-year-olds ( 9 girls; Median $=2$ years 1 month 3 days; Range $=2$ years 0 month 5 days to 2 years 6 months 15 days), a third sample of 392 -year-olds (19 boys; Median $=2$ years 0 months 26 days; Range $=2$ years 0 months 2 days to 2 years 2 months 0 days), a fourth sample of 183 -year-olds ( 8 girls; Median $=3$ years 2 months 15 days; Range $=3$ years 1 months 6 days to 3 years 5 months 18 days), and a fifth sample of 95 -year-olds ( 3 boys; Median $=5$ years 3 months 15 days; Range $=5$ years 0 months 11 days to 5 years 6 months 10 days) were tested. Children were recruited from a local database of a midsized German city (population approximately 500,000; median household monthly income approximately 1400€) and accompanied by their parents who gave informed consent prior to participating. All children were native German speakers and predominately White Caucasian. Children received a small toy for participation.

From the original samples, we included those children for whom we obtained information regarding their siblings, whether or not they visited daycare, and the area of the city they lived in. Parents provided this information on a voluntary basis. In addition we only included those subjects for whom we obtained data both during the baseline measurement as well as the process measurement phase (see details below). Therefore the sample sizes for the present analyzes are smaller than those reported in the original studies (see Table 1 for details).

\subsection{Materials and design}

Children sat in front of a house apparatus. On the child's side a small 
window was cut into the frame, such that it fit a computer screen (see Fig. 1 for an illustration). A webcam attached to the other side of the apparatus provided a live feed to the computer screen children were facing. A mobile eye-tracking unit (Tobii Model $\times 120$ or SMI model red-m) recorded participants' pupil diameter.

From each study sample we included only the first test trial to capture children's spontaneous response to seeing the adult needing help. In each study, the test trial followed the identical sequence of events: (1) a baseline measure of pupil dilation, (2) live-feed showing an adult needing help, (3) process measure of pupil dilation, and (4) an opportunity for the child to immediately help the adult. The actual measurement of internal arousal, i.e., pupil dilation, was taken while a neutral stimulus was presented on the computer screen during the baseline and process measurement phases (see Hepach et al., 2016ba). This stimulus consisted of computer-animated bubbles (total duration $11 \mathrm{~s}$ ) and was preceded by a blinking colored dot to center children's attention.

\subsection{Procedure}

During the study, children either (1) sat on their parent's lap (samples 1-3), (2) sat in front of the house while the parent sat on a separate chair pretending to read a magazine (sample 4), or (3) sat in front of the house while their parent sat in an adjacent room and watched the study through a one-way mirror (sample 5). In cases where parents accompanied their child to the study room, they were asked not to provide any cues during the study. In the following we describe the commonalities in the procedures between the separate samples. For procedural details please refer to the original studies (see Table 1).

A female experimenter (E1) accompanied all children and guided them through the study. Upon entering the study room, children sat in front of the computer screen and watched an animated object move to five pre-defined locations (standard calibration procedure; see also Gredebäck et al., 2010). A second adult experimenter (E2) moved to the inside of the house such that he (samples 3-5) or she (samples 1-2) was captured by the live-feed. All children were first familiarized to the house apparatus and witnessed E2 move back and forth between the 'inside' and 'outside' of the house to ensure that they understood the scene on the computer screen to resemble the actual events on the other side of the apparatus (see also Hepach et al., 2012; Troseth \& de Loache, 1998). After the familiarization, children moved in front of the computer screen. A third adult (E3) was in charge of running the computer software and presentation of stimuli from a separate room. Through an intercom headset this adult communicated with the adults inside the study room. This was crucial for the timing of events (see details below).

The actual study began with E2 moving to the inside of the house and children sitting on the outside in front of the computer screen (see Fig. 1). Children were then presented with the first neutral stimulus (baseline measure). Next children observed, through the video livefeed, E2 carrying out a task. This activity was different for each age group in order to keep children similarly engaged in age-appropriate situations. After a predetermined period of time the adult encountered an instrumental problem and was forced to interrupt his/her activity (see Table 1 for details). Thus, all children saw the adult experimenter needing help.

At this point, for all age groups, the neutral stimulus was presented for a second time (process measure of pupil dilation). Subsequently, parents carried their child to the inside of the house (18- and 24-montholds, samples 1-3) or children walked over to the other side (3- and 5year-olds, samples 4-5). E2 incrementally increased her cues for help in case children did not immediately respond (see also Svetlova et al., 2010). For samples 1 and 2, 18-month-olds and 24-month-olds saw the adult reaching for the object (10 s), saying 'Oh, I can't reach it' (5 s), 'I need help' ( $5 \mathrm{~s})$, 'I need help [child's name] ( $5 \mathrm{~s}$ ), and explicitly asking the child for help (5 s). For sample 3, 24-month-olds saw E2 reaching 

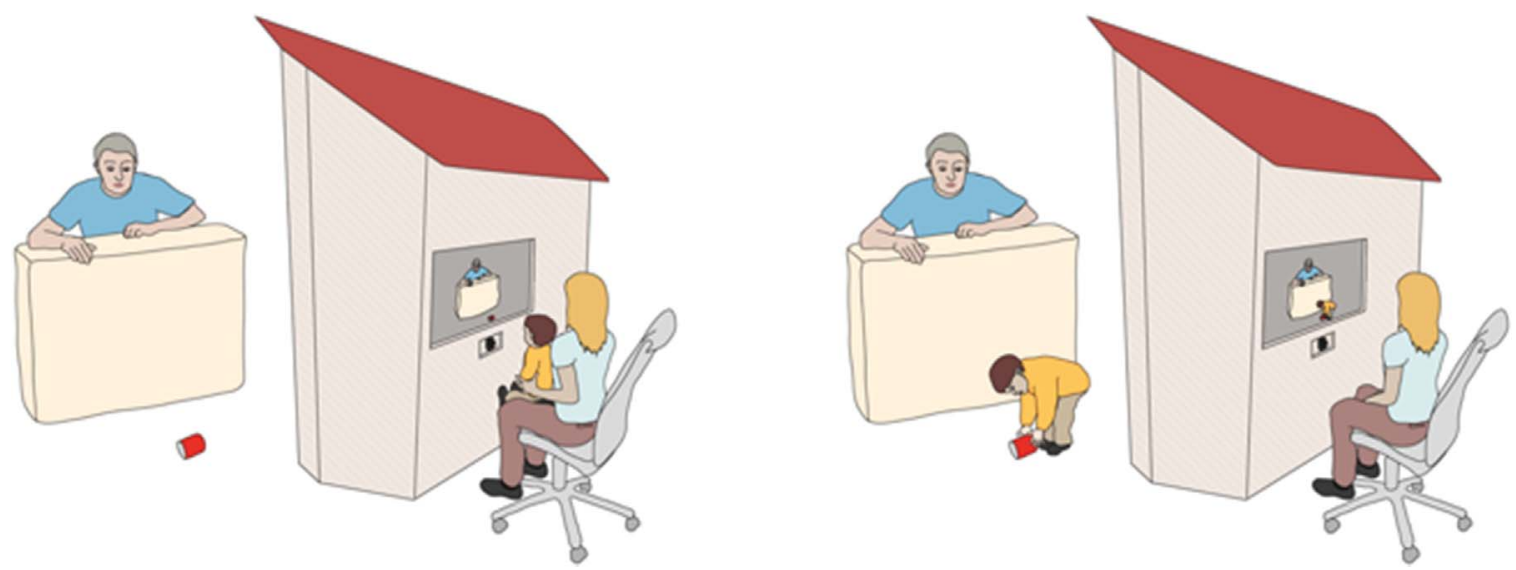

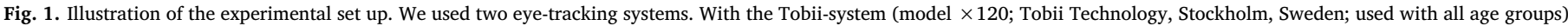

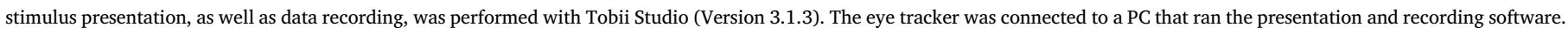

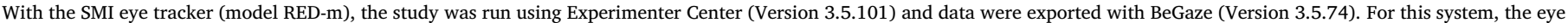
tracker was connected to a separate laptop that was in turn connected to the main PC running Experiment Center and BeGaze.

for the object (7 s), alternating gaze while reaching ( $8 \mathrm{~s})$, addressing the child and directing her attention to the dropped object $(15 \mathrm{~s})$, and asking the child 'Can you help me?' (15 s). Not all 18- and 24-montholds in these samples helped the adult. For those who did not provide help, E2 stopped reaching after $30 \mathrm{~s}$ (samples 1-2) or $45 \mathrm{~s}$ (sample 3) and the trial ended. For samples 4 and 5, 3- and 5-year-old children saw E2 increasing his cues for help by first stating that a liquid had spilled and briefly looking at the child (approx. $10 \mathrm{~s}$ ), saying that he needed the paper towel to wipe up the spilled liquid (approx. $5 \mathrm{~s}$ ), and explicitly asking the child to hand him the paper towel until they did so. Thus, the designs for samples 4-5 were such that all 3-and 5-year-olds provided help; they thus differed only in their latency to do so. In every study, once the test procedure was completed, all children were given a chance to help the adult and to see the situation resolved.

\subsection{Data analysis}

The pre-processing of pupil data followed that of Hepach et al. (2012) (see also Hepach et al., 2013, 2016a). Each presentation of the neutral stimulus (once during the baseline measurement and once during the process measurement) elicited two pupillary light reflexes in brief succession. The two minima of these light reflexes were identified to assess the degree of pupil dilation (see also Henderson et al., 2014; Steinhauer et al., 2000, for a similar analysis). In cases where data for both reflexes were obtained, the average was computed. Otherwise we used the data point for the single pupillary minimum available. This was done for both the baseline and process measure of pupil dilation. For each child, we were specifically interested in the change from the baseline to the process measure in pupil dilation given that this captured their immediate response to seeing the adult needing help. In addition, we included in our analyses children's baseline measure of pupil dilation as an indicator of their tonic state arousal at the beginning of the study.

We recorded the time in seconds from the moment the children were placed on a specified mark on the floor by their parents (samples 1-3) or once children got up to walk to E1's side (samples 4 and 5), until they attempted to pick up the needed out-of-reach object. Ten children initiated their helping behaviour toward the end of the test trial and their latency to hand over the object was therefore greater than the maximum latency for the non-helpers. This was controlled for by the statistical analyses (see details below).

The statistical significance of both the change in pupil dilation as well as the baseline level of pupil dilation was investigated through conducting survival analyses (also referred to as hazard function analyses) in $R$ (Therneau and Grambsch, 2000) using the packages 'survival' (Therneau, 2015) and 'coxme' (Therneau, 2015b). In survival analyses the dependent measure comprises both the information of whether children helped and if they helped, how fast they were to do so (the latency in s). More specifically, the dependent measure per subject reflects the proportion of subjects who helped later than the respective subject. Over time (latency in s) this proportion of 'not-yet-helpers' becomes smaller as the likelihood for children to eventually help increases with time (note that all children in the older age groups helped, see Table 1).

Survival analyses provide a crucial advantage over other modelling techniques. In every study researchers determine a maximum time they allow for children to help. In survival analyses children who did not help within this arbitrarily set time window are not treated as 'not having helped' but rather as 'not having helped yet'. This allows for the inclusion of data drawn from separate studies with varying maximum time windows defined for children's helping behaviour to occur. Therefore, running survival analyses provides an adequate modelling strategy to include both those children who helped and those children who did not help across several data sets.

Similar to other modelling techniques, the statistical significance of predictors is determined through model comparisons. The full regression model included both the baseline measure of tonic arousal state of pupil dilation as well as the change in pupil dilation. In addition, we included the following control variables: gender, siblings (yes or no), whether the child attended day care (yes or no), age group (1, 2, 3, or 5 years) and age normalized within each age group. We chose this coding of age for the following reasons. Although the order and the nature of events children saw was similar within each study, the specific procedures were adapted to meet the attentional demands of each age group (see Table 1 for details). It is important to include age group in the model but a statistically significant effect may reflect a difference between study procedures instead of a genuine development trend. At the same time, we sought to investigate an effect of age within each age group. Instead of using children's age in days (which includes the information of age group) we normalized age in days within each age group to a minimum of 0 and to a maximum of 1 . This made the exact ages within each age group comparable across all age groups. We further square-root-transformed the normalized variable to achieve normal distribution. In addition, societal milieu was included as a random effect. We first determined the district that children and their families lived in (see also Helbig, 2010). Based on the city's census data, the socio-demographic information of age (mode), education (graduation and kind of apprenticeship, average), relationship status (average), net income (individual and for the household, median) and percentages of retirees and unemployed persons (average), every district (and thus 
every subject) was assigned to a societal milieu (Sinus Sociovision, 2014).

The statistical significance of both measures of pupil dilation was established through comparing the full model to a reduced model (one without the change in pupil dilation and one without the baseline measure of pupil dilation) including only the control factors and random effect. The statistical significance of the control factors gender, siblings, and age was investigated through their respective regression coefficients in the full model. Cox regression models provide effect ratios for each predictor variable. For a dichotomous categorical predictor, a ratio greater than 1 indicates a positive effect and a ratio smaller than 1 indicates a negative effect on children's likelihood to help. Finally, the statistical contribution of societal milieu was established through comparing the full model to a reduced model without the random effect of milieu.

\section{Results}

Overall, 81 out of 109 (approx. 74\%) children helped (see Table 1 for details regarding the individual studies). There was no difference between boys ( 44 out of $55,80 \%$ ) and girls ( 37 out of $54,69 \%$ ) in their rates of helping, $\chi^{2}(1)=1.33, p=.25$. For those children who helped, the average latency to help was $15.63 \mathrm{~s}(S D=13.31)$. There was no difference in the latency to help between boys $(M=15.61, S D=$ $14.02)$ and girls $(M=15.65, S D=12.6)$, Mann-Whitney- $U$ (exact $)=$ $764, p=.64$.

As a preliminary analysis, we ran a linear regression to assess whether any of the control variables (sibling, daycare attendance, gender and age) as well as children's baseline pupil dilation had an effect on the change in children's pupil dilation. This did not reveal statistically significant effects of gender $(p=.26)$, day care attendance ( $p=.3$ ), or age group $(p=.28$ ) but did reveal statistically significant effects for growing up with a sibling $(\beta=.04+/-.03, p=.04)$, an effect of age within age groups $(\beta=.07+/-.04, p=.049)$, and an effect of children's baseline measure of pupil dilation such that higher baseline levels of tonic arousal were related to smaller changes in pupil dilation $(\beta=-.05+/-.01, p<.001)$. Given this relation between children's baseline pupil dilation and their change in pupil dilation we investigated their respective effects on children's helping in two separate analyses.

The main analysis revealed that children's propensity to help was influenced by the change in children's pupil dilation (baseline-corrected). The greater the change in children's pupil dilation in response to seeing the adult in need of help, the more likely and faster they were to provide the help, $\beta=.04+/-.02$, effect ratio $=1.04, \chi^{2}(1)=$ $5.67, p=.02$ (see Fig. 2). An additional correlation analysis including only those children who helped did not yield a statistically significant relation between the change in pupil dilation and the latency to provide the help, $r$ (Pearson) $=-.04, p=.72$.

On the other hand, there was no statistically significant effect of children's baseline measure of pupil dilation and their likelihood to help, $\beta=.36+/-.22, \chi^{2}(1)=2.82, p=.09$. In addition, there were no effects of gender $(z=1.03, p=.3)$, day care attendance $(z=.09, p$ $=.93)$, growing up with a sibling $(z=-1.77, p=.08)$, or age in within age group $(z=.99, p=.32)$. The analysis did reveal an effect of age group, $\beta=.56+/-.13$, effect ratio $=1.74, z=4.29, p<.001$.
Yes
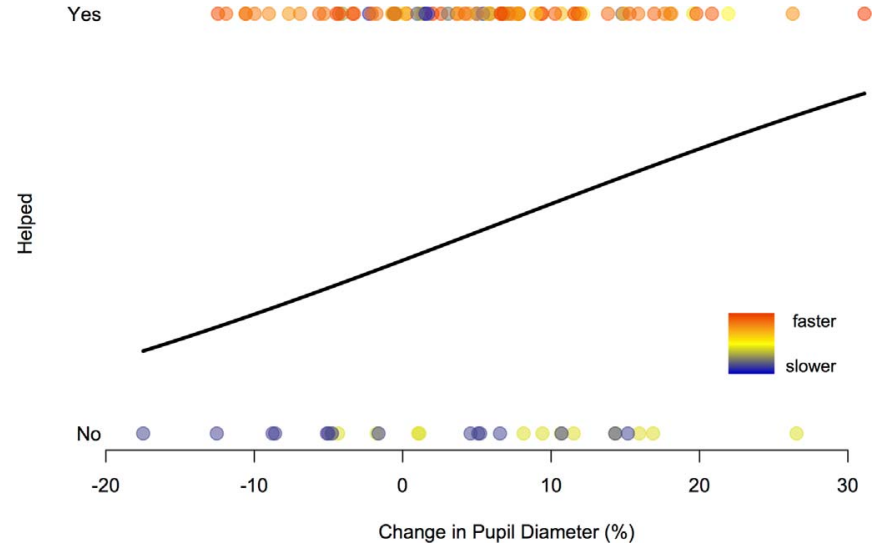

Fig. 3. The data and model line for the main statistical analysis. The solid center line shows the modeled change in pupil dilation. The $\mathrm{x}$-axis represents the change in pupil dilation from baseline (at the beginning of the test trial) to immediately after children saw the adult needing help but just before they had the opportunity to help. Negative values indicate that children's pupil dilation before helping was lower than their initial baseline level of internal arousal. The greater children's change in pupil dilation, the more likely they were to help the adult.

Societal milieu had no statistical influence on children's propensity to help, $\chi^{2}(1)=6.14, p=.52$

\section{Discussion}

Although helping emerges early in ontogeny, young children vary in their motivation to help others and little is known about the motivation, in particular changes in physiological arousal, underlying children's prosocial behaviour. Here, we showed that children's motivation to help, from 18-month-old toddlers to 5-year-old preschoolers, is related to the degree of internal arousal that children experience when they see a person in need of help. Importantly, it was the baseline-corrected degree of change in children's pupil dilation in response to the helping situation - and not the baseline measure of children's pupil dilation that was positively related to their helping. This suggests that changes in children's pupil dilation directly reflect their motivation to help. The greater the increase in their internal physiological arousal in response to seeing others needing help, the faster and more likely children are to help (Fig. 3).

These results suggest that assessing children's internal arousal taps into their intrinsic motivation to help, which is in line with previous work on children's helping behaviour, some of which demonstrated that for children up to an age of 3 years change in pupil dilation cannot be attributed soley to them seeking approval by an adult but rather reflect a motivation to see the adult in need be helped (Hepach et al., 2012, 2016b; see also Ulber et al., 2016; Warneken and Tomasello, 2008 for a similar interpretation). However, this interpretation of intrinsic motivation needs to be further qualified by future research. Note that in the studies included in the present analyses the adult increased his/her cues for help, making the need incrementally more explicit thus providing an extrinsic incentive (i.e., adult approval) for helping. Research on the impact of adult praise on children's helping behaviour is mixed. While some controlled experimental studies have found no effects of parental encouragement or adult praise on children's helping (Warneken and

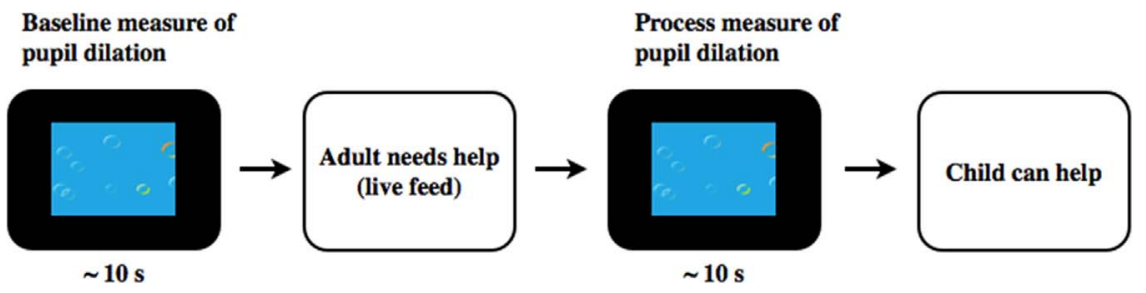

Fig. 2. The order of events within each study. 
Tomasello, 2008, 2012) other studies found increases in young children's helping following praise (Dahl et al., 2017). These results raise a relevant question for future research, i.e., to what degree changes in children's internal arousal in response to seeing others in need are a socially scaffolded response.

Previous work had found that increases in children's physiological arousal, as indicated by increased heart rate, were related to reduced willingness to help (e.g., Eisenberg et al., 1988b, 1996). However, those studies had only indirectly investigated the relation between children's internal arousal and their tendency to be prosocial by collecting the physiological measures in response to video stimuli (see Hastings et al., 2006, for a review). Our results suggest that children's internal arousal relates to their propensity to help. This would suggest that the physiological changes recorded in the present set of studies may be comparable to the cardiac slowing which has been linked to increased prosocial behaviour (Eisenberg et al., 1988b; Eisenberg et al., 1989; Eisenberg and Fabes, 1990; see also Libby et al., 1973, for a similar relation). More research, possibly co-assessing heart rate and pupil dilation changes in the same experimental paradigm, is needed to further qualify the physiological mechanism as part of the emotions underlying children's prosocial behaviour.

Furthermore, in the present investigation, age was positively related to children's propensity to help the adult. Although this result might reflect a genuine developmental change, it is important to note that it may also be a consequence of the experimental manipulation. Future studies, potentially using longitudinal designs, should more systematically investigate age as a factor in children's instrumental helping (see also Baillargeon et al., 2011; Hay et al., 1999; Zahn-Waxler et al., 1992). An additional question for future work is whether at an early age, children's diminished responses to others in need (i.e., small change in internal arousal) are related to inhibited prosocial or disruptive behaviour, which might become more evident in later ontogeny with a longitudinal design (Hay et al., 1999; Young et al., 1999).

We focused our investigation on the relation between children's internal arousal and their motivation to help in the context of instrumental helping behaviour. The relation between these two variables may be less direct with other forms of helping behaviour where the correct means to help are not as readily identifiable, e.g., comforting behaviour (see also Dunfield, 2014). Moreover, even within the same domain of helping behaviour, children's motivation to help may change across development (Eisenberg and Spinrad, 2014; Jensen et al., 2014; Warneken and Tomasello, 2009). Future research needs to investigate the association between children's internal arousal and helping in different tasks and contexts. In addition, one finding of the present analyses suggests that children who grow up with at least one sibling showed greater increase in pupil dilation to response to seeing an adult needing help. We included the sibling information in our analyses because prior work showed that sibling relationship relates to children's individual differences in helping behaviour (Brownell, Zerwas, \& Balaraman, 2002; Ross, 1996). At the same time, prior work also suggests that the influence of growing up with a sibling depends on additional factors such as the age of the sibling (e.g., Hay et al., 1999). Given that we did not have detailed data on the number and age of the siblings, the conclusions we can draw from the present data set are limited and more work is needed to investigate how sibling relationships affect children's prosocial behaviour. One possible avenue for future research is to more systematically investigate the influence of social context variables both more proximate (observers present or encouragement given) and more distant (siblings and parenting style) on children's tonic baseline internal arousal, the arousal response to seeing others in need, and on their actual helping behaviour. It is conceivable that such social variables influence children's internal arousal and thus predict their helping behaviour, similar to the influence of other socio-cognitive variables such as joint attention skills and self-other differentiation (Kärtner et al., 2014) as well as socialization practices (Giner Torréns and Kärtner, 2017) on children's prosocial behaviour.
The data comprised in the current analyses reflected changes in children' tonic arousal state, which are different from phasic changes. The latter reflect relatively fast stimulus-evoked changes in pupil dilation whereas tonic pupil dilation is commonly measured by averaging pupil diameter over at least several seconds (Beatty and LuceroWagoner, 2000; Granholm and Steinhauer, 2004). While it is therefore possible that the increased attentional demands of seeing a person needing help affect children's changes in pupil dilation, additional studies are needed that include measures of phasic changes and can isolate the contribution of mental effort to children's changes in pupil dilation in response to seeing others needing help.

It is important to note that the present investigation addressed the link between children's physiological responsiveness to seeing an individual needing help and the likelihood of their actual helping behaviour. Investigating the exact nature of the mechanistic role of these physiological changes in children's intention formation and behaviour execution is the necessary next step in future research. One approach is to include more measurement points of pupil dilation, e.g., at the beginning of the trial, after seeing another needing help, and after seeing the situation being resolved. An analysis of two-year-old children's changes in pupil dilation across the three time points revealed that the more children's pupil dilation increased to seeing an adult needing help, the more it remained high if no appropriate help was provided and the more pupil dilation decreased if children saw the appropriate help being provided. Crucially this was not the case in a non-social control scenario in which no human agents were present and no need for help arose (Hepach et al., 2016a). A complementary approach for future research is to measure physiological changes more immediately within the situation using wearable eye-trackers (e.g., Kretch and Adolph, 2016; Smith et al., 2015) though effects of luminosity need to be taken into consideration when assessing changes in children's pupil dilation. A further possible avenue for future research is to combine the measure of pupillary changes with other physiological variables, such as blood pressure (Gendolla, 1998) or neurophysiological variables of approachavoidance motivation (Fox, 1991; Harmon-Jones, 2003).

The present data set comprised studies that included one type of experimental condition, namely children saw an adult needing help. The fact that changes in children's internal arousal related to whether and how fast children subsequently helped the adult raises important questions for future research. First, are the changes in pupil dilation specific to seeing the need of another fulfilled or does it reflect a more general motivation to execute a behaviour? Addressing this question necessitates the inclusion of a control condition, one that does not constitute a helping situation. One approach has been taken by Hepach et al. (2016a), in which children were presented with either a social need condition (an adult dropped object while working on a task) or non-social control condition (objects dropped without an agent present). In this eye-tracking study children showed more involvement in the social condition by demonstrating more anticipatory gaze behaviour and greater increase in pupil dilation compared to the non-social condition. In addition, the authors measured pupil dilation one additional time at the end of the trial when the situation was either resolved congruently or incongruently (see Hepach et al., 2016a, for details). The inclusion of this additional measurement point revealed the time course of the changes in children's pupil dilation: The greater children's pupil dilation in response to seeing the needs the more it subsided if the adult's need was congruently fulfilled and the more it remained increased if the adult was not helped. No such pattern emerged in the non-social control condition. Based on these results of previous work one interesting avenue for future research is the inclusion of a nonsocial control condition in a live behavioural paradigm. One question then is whether children's pupil dilation increases less in a non-social control condition. A second related question is whether the relation between the change in children's pupil dilation and their likelihood to help is weaker in a non-social control condition.

In sum, from 18 months to 5 years of age, changes in children's 
internal arousal as measured in their pupil dilation are linked to their motivation to fulfill others' needs. Individual differences in children's helping can thus be traced, at least in part, to individual differences in children's physiological and motivational involvement upon seeing others in need.

\section{References}

Baillargeon, R.H., Morisset, A., Keenan, K., Normand, C.L., Jeyaganth, S., Boivin, M., Tremblay, R.E., 2011. The development of prosocial behaviors in young children: a prospective population-based cohort study. J. Genet. Psychol. 172 (3), 221-251.

Beatty, J., Lucero-Wagoner, B., 2000. The pupillary system. In: Cacioppo, J.T., Tassinary, L.G., Berntson, G.G. (Eds.), Handbook of psychophysiology, 2nd ed. Cambridge University Press, New York, NY, pp. 142-162.

Bischof-Köhler, D., 1991. The development of empathy in infants. Infant Dev.: Perspect. Ger.-Speak. Ctries. 245-273.

Bradley, M.M., Miccoli, L., Escrig, M.A., Lang, P.J., 2008. The pupil as a measure of emotional arousal and autonomic activation. Psychophysiology 45, 602-607.

Brehm, J.W., Self, E.A., 1989. The intensity of motivation. Annu. Rev. Psychol. 40 (1), 109-131.

Brooks-Gunn, J., Duncan, G.J., Klebanov, P.K., Sealand, N., 1993. Do neighborhoods influence child and adolescent development? Am. J. Sociol. 353-395.

Clarke-Stewart, A., 2010. A home is not a school: the effects of child care on children's development. J. Social. Issues 47 (2), 105-123.

Dahl, A., Satlof-Bedrick, E.S., Hammond, S.I., Drummond, J.K., Waugh, W.E., Brownell, C.A., 2017. Explicit scaffolding increases simple helping in younger infants. Dev. Psychol. 53 (3), 407.

Dunfield, K., Kuhlmeier, V.A., O'Connell, L., Kelley, E., 2011. Examining the diversity of prosocial behavior: helping, sharing, and comforting in infancy. Infancy 16 (3), $227-247$.

Dunfield, K.A., Kuhlmeier, V.A., 2013. Classifying prosocial behavior: children's responses to instrumental need, emotional distress, and material desire. Child Dev. 84 (5), $1766-1776$.

Dunfield, K.A., 2014. A construct divided: prosocial behavior as helping, sharing, and comforting subtypes. Front. Psychol. 5.

Dunn, J., Munn, P., 1986. Siblings and the development of prosocial behaviour. Int. J. Behav. Dev. 9 (3), 265-284.

Eisenberg, N., Fabes, R.A., Bustamante, D., Mathy, R.M., Miller, P.A., Lindholm, E., 1988a. Differentiation of vicariously induced emotional reactions in children. Dev. Psychol. 24 (2), 237.

Eisenberg, N., McCreath, H., Ahn, R., 1988b. Vicarious emotional responsiveness and prosocial behavior: their interrelations in young children. Personal. Social. Psychol. Bull. 14 (2), 298-311.

Eisenberg, N., Fabes, R.A., Miller, P.A., Fultz, J., Shell, R., Mathy, R.M., Reno, R.R., 1989. Relation of sympathy and personal distress to prosocial behavior: a multimethod study. J. Personal. Social. Psychol. 57 (1), 55.

Eisenberg, N., Fabes, R., 1990. Empathy: conceptualization, measurement, and relation to prosocial behavior. Motiv. Emot. 14 (2), 131-149.

Eisenberg, N., Spinrad, T.L., 2014. Multidimensionality of Prosocial Behavior. In: PadillaWalker, Laura M., Carlo, Gustavo (Eds.), Prosocial development: A multidimensional approach. Oxford University Press, pp. 17-39.

Fox, N.A., 1991. If it's not left, it's right: electroencephalograph asymmetry and the development of emotion. Am. Psychol. 46 (8), 863.

Gendolla, G.H., 1998. Effort as assessed by motivational arousal in identity-relevant tasks. Basic Appl. Social. Psychol. 20 (2), 111-121.

Giner Torréns, M., Kärtner, J., 2017. The influence of socialization on early helping from a cross-cultural perspective. J. Cross-Cult. Psychol. 48 (3), 353-368.

Granholm, E., Steinhauer, S.R., 2004. Pupillometric measures of cognitive and emotional processes. Int. J. Psychophysiol. 52 (1), 1-6.

Gredebäck, G., Johnson, S., von Hofsten, C., 2010. Eye tracking in infancy research. Dev. Neuropsychol. 35, 1-9.

Grossmann, T., Johnson, M.H., 2007. The development of the social brain in human infancy. Eur. J. Neurosci. 25 (4), 909-919.

Harmon-Jones, E., 2003. Clarifying the emotive functions of asymmetrical frontal cortical activity. Psychophysiology 40 (6), 838-848.

Harper, J.M., Padilla-Walker, L.M., Jensen, A.C., 2014. Do siblings matter independent of both parents and friends? Sympathy as a mediator between siblings relationship quality and adolescent outcomes. J. Res. Adolesc. 1-14.

Hastings, P.D., Zahn-Waxler, C., McShane, K., 2006. We are, by nature, moral creatures: biological bases of concern for others. In: Killen, M., Smetana, J.G. (Eds.), Handbook of moral development. Erlbaum, Mahwah, NJ, pp. 483-516.

Hay, D.F., Castle, J., Davies, L., Demetriou, H., Stimson, C.A., 1999. Prosocial action in very early childhood. J. Child Psychol. Psychiatry 40 (6), 905-916.

Helbig, M., 2010. Neighborhood does matter! Soziostrukturelle Nachbarschaftscharakteristika und Bildungserfolg. Kölner Z. Soziol.Soz. 62 (4), 655-679.

Henderson, R.R., Bradley, M.M., Lang, P.J., 2014. Modulation of the initial light reflex during affective picture viewing. Psychophysiology 51, 815-818.

Hepach, R., Vaish, A., Tomasello, M., 2012. Young children are intrinsically motivated to see others helped. Psychol. Sci. 23 (9), 967-972.

Hepach, R., Vaish, A., Tomasello, M., 2013. A new look at children's prosocial motivation. Infancy 18 (1), 67-90.

Hepach, R., Westermann, G., 2016. Pupillometry in infancy research. J. Cogn. Dev. 17 (3), 359-377.

Hepach, R., Vaish, A., Grossmann, T., Tomasello, M., 2016a. Young children want to see others get the help they need. Child Dev. 87 (6), 1703-1714.
Hepach, R., Vaish, A., Tomasello, M., 2016b. Children's intrinsic motivation to provide help themselves after accidentally harming others. Child Dev.

Jencks, C., Mayer, S.E., 1990. The social consequences of growing up in poor neighborhood. In: Lynn, L.E., MyGeary, M.G.H. (Eds.), Inner-city poverty in the United States. National Academy Press, Washington, DC, pp. 111-186.

Jensen, K., Vaish, A., Schmidt, M.F., 2014. The emergence of human prosociality: aligning with others through feelings, concerns, and norms. Front. Psychol. 5.

Kärtner, J., Schuhmacher, N., Collard, J., 2014. Socio-cognitive influences on the domainspecificity of prosocial behavior in the second year. Infant Behav. Dev. 37 (4), 665-675.

Kasten, H., 2007. Einzelkinder und ihre Familien. Hogrefe: Göttingen, Germany.

Köster, M., Ohmer, X., Nguyen, T.D., Kärtner, J., 2016. Infants understand others' needs. Psychol. Sci. 27 (4), 542-548. http://dx.doi.org/10.1177/0956797615627426.

Kretch, K.S., Adolph, K.E., 2016. The organization of exploratory behaviors in infant locomotor planning. Dev. Sci.

Laeng, B., Sirois, S., Gredebäck, G., 2012. Pupillometry a window to the preconscious? Perspect. Psychol. Sci. 7 (1), 18-27.

Lenzi, M., Vieno, A., Perkins, D.D., Pastore, M., Santinello, M., Mazzardis, S., 2012. Perceived neighborhoods social resources as determinants of prosocial behavior in early adolescence. Am. J. Community Psychol. 50, 37-49.

Libby, W.L., Lacey, B.C., Lacey, J.I., 1973. Pupillary and cardiac activity during visual attention. Psychophysiology 10 (3), 270-294.

Liebenwein, S., 2008. Erziehung und soziale Milieus: elterliche Erziehungsstile in milieuspezifischer Differenzierung. Springer, Wiesbaden.

Loewenfeld, I.E., 1993. The Pupil: Anatomy, Physiology, and Clinical Applications. Wayne State University Press, Detroit, MI.

Martin, A., Olson, K.R., 2015. Beyond good and evil: what motivations underlie children's prosocial behavior? Perspect. Psychol. Sci. 10 (2), 159-175.

Miller, P., Eisenberg, N., Fabes, R., Shell, R., 1996. Relations of moral reasoning and vicarious emotion to young children's prosocial behavior toward peers and adults. Dev. Psychol. 32 (2), 210.

Paulus, M., 2014. The emergence of prosocial behavior: why do infants and toddlers help, comfort, and share? Child Dev. Perspect. 8 (2), 77-81.

Reid, V.M., Hoehl, S., Grigutsch, M., Groendahl, A., Parise, E., Striano, T., 2009. The neural correlates of infant and adult goal prediction: evidence for semantic processing systems. Dev. Psychol. 45 (3), 620.

Rheingold, H.L., Hay, D.F., 1980. Prosocial behavior of the very young. Moral. a Biol. Phenom. 93-108.

Richter, M., Gendolla, G.H.E., Wright, R.A., 2016. Three decades of research on motivational intensity theory: what we have learned about effort and what we still don't know. Adv. Motiv. Sci. 3, 149-168.

Romano, E., Tremblay, R.E., Boulerice, B., Swisher, R., 2005. Multilevel correlates of childhood physical aggression and prosocial behaviour. J. Abnorm. Child Psychol. $33,565-578$.

Ross, H.S., 1996. Negotiating principles of entitlement in sibling property disputes. Dev. Psychol. 32 (1), 90.

Roth-Hanania, R., Davidov, M., Zahn-Waxler, C., 2011. Empathy development from 8 to 16 months: early signs of concern for others. Infant Behav. Dev. 34 (3), 447-458.

Sarafino, E.P., 1985. Peer-peer interaction among infants and toddlers with extensive daycare experience. J. Appl. Dev. Psychol. 6 (1), 17-29.

Schmidt-Denter, U., 2005. Soziale Beziehungen im Lebenslauf. Beltz: Weinheim.

Sinus Sociovision, 2014. December 17). Sinus-Milieus. Retrieved from 〈http://www. sinus-institut.de/loesungen/sinus-milieus.html>.

Sirois, S., Brisson, J., 2014. Pupillometry. Wiley Interdiscip. Rev.: Cogn. Sci. 5 (6), 679-692.

Smith, L.B., Yu, C., Yoshida, H., Fausey, C.M., 2015. Contributions of head-mounted cameras to studying the visual environments of infants and young children. J. Cogn. Dev. 16 (3), 407-419.

Steinhauer, S.R., Condray, R., Kasparek, A., 2000. Cognitive modulation of midbrain function: task-induced reduction of the pupillary light reflex. Int. J. Psychophysiol. 39 (1), 21-39.

Svetlova, M., Nichols, S.R., Brownell, C.A., 2010. Toddlers' prosocial behavior: from instrumental to empathic to altruistic helping. Child Dev. 81 (6), 1814-1827.

Therneau, T.M., 2015a. A Package for Survival Analysis in S. version 2.38, 〈https:// CRAN.R-project.org/package $=$ survival $>$.

Therneau, T.M., 2015b. coxme: Mixed Effects Cox Models. R package version 2.2-5. $\langle$ https://CRAN.R-project.org/package = coxme $\rangle$.

Therneau, T.M., Grambsch, P.M., 2000. Modeling Survival Data: xtending the Cox Model. Springer, New York.

Ulber, J., Hamann, K., Tomasello, M., 2016. Extrinsic rewards diminish costly sharing in 3-year-olds. Child Dev. 87 (4), 1192-1203.

Warneken, F., Tomasello, M., 2008. Extrinsic rewards undermine altruistic tendencies in 20-month-olds. Dev. Psychol. 44, 1785-1788.

Warneken, F., Tomasello, M., 2009. Varieties of altruism in children and chimpanzees. Trends Cogn. Sci. 13 (9), 397-402.

Warneken, F., 2015. Precocious prosociality: why do young children help? Child Dev. Perspect. 9 (1), 1-6.

Woodward, A.L., 2009. Infants' grasp of others' intentions. Curr. Dir. Psychol. Sci. 18, 53-57. http://dx.doi.org/10.1111/j.1467-8721.2009.01605.x.

Wright, R.A., Kirby, L.D., 2001. Effort determination of cardiovascular response: an integrative analysis with applications in social psychology. Adv. Exp. Social. Psychol. $33,255-307$.

Young, S.K., Fox, N.A., Zahn-Waxler, C., 1999. The relations between temperament and empathy in 2-year-olds. Dev. Psychol. 35 (5), 1189.

Zahn-Waxler, C., Radke-Yarrow, M., Kind, R.A., 1979. Child rearing and children's prosocial initations toward victims of distress. Child Dev. 50, 319-330.

Zahn-Waxler, C., Radke-Yarrow, M., Wagner, E., Chapman, M., 1992. Development of concern for others. Dev. Psychol. 28 (1), 126. 